\title{
MFSK Signal Individual I dentification Algorithm Based on Bi-spectrum and Wavelet Analyses
}

\author{
Fang $\mathrm{Ye}^{1}$, Jie Chen ${ }^{1}$, Yibing $\mathrm{Li}^{1}$, and Juan $\mathrm{Ge}^{2}$ \\ ${ }^{1}$ College of Information and Communication Engineering, \\ Harbin Engineering University, Harbin, China \\ [e-mail: yefang0815@sina.cn, sandra@hrbeu.edu.cn, liyibing0920@sina.cn] \\ ${ }^{2}$ Southwest China Research Institute of Electronic Equipment, \\ Chengdu, China \\ [e-mail: cjadata@163.com] \\ *Corresponding author: Yibing Li
}

Received March 28, 2016; revised July 15, 2016; accepted August 7, 2016; published October 31, 2016

\begin{abstract}
Signal individual reconnaissance and identification is an extremely important research topic in non-cooperative domains such as electronic countermeasures and intelligence reconnaissance. Facing the characteristics of the complexity and changeability of current communication environment, how to realize radiation source signal individual identification under the low SNR conditions is an emphasis of research. A novel emitter individual identification method combined bi-spectrum analysis with wavelet feature is presented in this paper. It makes a feature fusion of bi-spectrum slice characteristics and energy variance characteristics of the secondary wavelet transform coefficient to identify MFSK signals under the low SNR (signal-to-noise ratios) environment. Theoretical analyses and computer simulation results show that the proposed algorithm has good recognition performance with the ability to suppress noise and interference, and reaches the recognition rate of more than $90 \%$ when the SNR is $-6 \mathrm{~dB}$.
\end{abstract}

Keywords: Individual identification, Bi-spectrum slice, Wavelet transform, Feature fusion, Low SNR environment

This paper includes a concrete bi-spectrum analysis and a specific wavelet low-frequency analysis of MFSK signals. Based on two feature extraction analyses of MFSK, the paper proposes a original identification algorithm for MFSK under low SNR. This research was supported by National Natural Science Foundation of China (Grant No. 51509049), the Natural Science Foundation of Heilongjiang Province China (Grant No. F201345), the Fundamental Research Funds for the Central Universities of China (No. GK2080260140), and the National key foundation for exploring scientific instrument of China (Grant No. 2016YFF0102806). 


\section{Introduction}

$\mathbf{W}_{\text {ith the rapid development of digital signal processing technology, the radiation source }}$ signal individual identification is facing more severe challenges in non-cooperative domains such as electronic countermeasures and intelligence reconnaissance. That is, the mission is to realize the identification of emitter signal types and individual of the same type emitter signal by using a little of priori knowledge under the low SNR (signal to noise ratio) condition, and simultaneously, meet the real-time requirements of actual engineering application [1]. FSK (frequency shift keying) signal is a typical signal type in radar and communication field, and the study and research of the characteristics of MFSK (multiple frequency shift keying) signal are always the hot spot. Therefore, MFSK signal individual identification has essential application value and research significance.

A lot of researchers have done a lot of research of FSK signal individual recognition. Typical process of FSK signal individual identification is divided into three steps: signal preprocessing, parameter estimation or characteristics analysis and recognition algorithm. From the existing literatures, MFSK signal recognition methods can be divided into 2 categories: methods based on the likelihood function estimation [2-5], and methods based on signal feature extraction [6-11]. Obviously, different recognition methods have a tradeoff between computational complexity and identification accuracy [12]. In order to meet the requirements of practical applications, researchers have been exploring new solutions to improve the effectiveness of individual identification on the basis of the current communication environment. In the aspect of feature extraction, O.A.Dobre proposed a method to identify MFSK signal by using the first order cyclostationary feature without estimating parameters such as timing signal, instantaneous frequency [13]. And the recognition rate achieves about $90 \%$ when the SNR is $-4 \mathrm{~dB}$. Zhang set the average polymerization degree of signal's instantaneous frequency as new feature [14], which needs to estimate signal's instantaneous frequency firstly. Li presented a classification algorithm based on wavelet and higher-order cumulants [15], whose experiments show that Gaussian white noise can be suppressed effectively by the analysis of extracted characteristics parameters. Reference [16] studied the singularity characteristics of wavelet modulus. Zeng investigated the time-frequency distribution characteristics of the intercepted signal to extract peak ratio and peak number of Hough transform as identification features [17]. The method achieves great identification results when the SNR is -4dB. Zhao utilized multi-fractal characteristics for modulation order identification of MFSK signal [18], and verified that the algorithm is unaffected by the $\alpha$ stable distribution noise and Gaussian noise.

On the one hand, according to above methods, it can be seen that higher order spectrum analysis is a very useful tool for non-stationary signal analysis and feature extraction [19-21], which has been used to MFSK signal recognition [22-23] in many researches. Theoretically, higher order spectrum method can not only completely inhibit Gaussian distribution noise and retain abundant phase information, but also improve the accuracy of signal processing. In a large number of high order spectrum analytic techniques, bi-spectrum is the lowest order method, which meets the real-time requirement of signal processing.

On the other hand, due to the characteristics of multi-resolution analysis of wavelet analysis, the signal can be divided into low frequency approximation and a series of high frequency details. Thus, wavelet analysis can show signal's detail information in different scales [24-26]. 
Although the Gaussian noise and interference can be completely inhibited by bi-spectrum analysis theoretically, the non-Gaussian noise is powerless. The existence of non-Gaussian noise will disturb or even submerge subtle features, which increases the difficulty of fine feature extraction.

Through the elaboration of the merits and drawbacks of bi-spectrum and wavelet analyses, we can conclude that single analytic method cannot achieve ideal MFSK signal individual identification under low SNR. In order to address this problem, we adopt the idea of information fusion and propose two-dimensional feature fusion algorithm based on above two recognition methods. And the proposed algorithm combines bi-spectrum slice characteristics and wavelet coefficients characteristics to identify MFSK signal under low SNR condition.

This paper is organized as follows. Firstly, Section 2 and Section 3 present the bi-spectrum feature extraction and the wavelet low frequency feature extraction method respectively. Then, Section 4 presents the feature fusion method under low SNR conditions, Section 5 reports experimental results and analysis. Finally, conclusions are presented in Section 6.

\section{Bi-spectrum Feature Extraction}

In the field of signal processing, the first order statistics and the second order statistics play an important role. But for many signals, especially nonlinear signal, the characteristics cannot be well detected and represented by the second order statistics due to the influence of the devices or transmission interferences. As higher order statistics has one important characteristic that signal's phase information can be reflected by the second order spectrum, it can be used to extract the subtle features of MFSK signal individuals.

\subsection{Introduction of bi-spectrum slice}

Assume that the observed data $x:\{x(1), x(2), \ldots, x(N)\}$ is a real random sequence $(N$ denotes sequence length), the probability density function of $x$ is $p(x)$. The corresponding characteristic function $\Phi(\omega)$ can be denoted as:

$$
\Phi(\omega)=\int_{-\infty}^{+\infty} p(x) e^{j \omega x} d x=E\left[e^{j \omega x}\right]
$$

And the first order characteristic function of $N$-dimensional random vector $\bar{X}=\left[x_{1}, x_{2}, \ldots x_{N}\right]^{T}$ is defined as:

$$
\Phi(\bar{\omega})=E\left\{e^{j \bar{\omega}^{T} \bar{x}}\right\}=E\left\{e^{j\left(\omega_{1} x_{1}+\omega_{2} x_{2}+\ldots+\omega_{N} x_{N}\right)}\right\}
$$

where, $\bar{\omega}^{T}=\left[\omega_{1}, \omega_{2}, \ldots, \omega_{N}\right]$.

Take the logarithm for formula (1) (2), and the second order characteristic functions of $x, \bar{x}$ are separately defined as:

$$
\begin{gathered}
\Psi(\omega)=\ln \Phi(\omega)=\ln E\left\{e^{j \omega x}\right\} \\
\Psi(\bar{\omega})=\ln \Phi(\bar{\omega})=\ln E\left\{e^{j \bar{\omega}^{T} \bar{x}}\right\}
\end{gathered}
$$

The $k$-order moment of $x$ is defined as follows.

$$
\mathrm{m}^{k}=\left.(-j)^{k} \frac{d^{k} \Phi(\omega)}{d \omega^{k}}\right|_{\omega=0}=E\left\{x^{k}\right\}
$$


Therefore, the $k$-order cumulant of $x$ is:

$$
c_{k}=\left.(-j)^{k} \frac{d^{k} \Psi(\omega)}{d \omega^{k}}\right|_{\omega=0}=(-j)^{k} \Psi^{k}(\omega)
$$

From formula (5) (6), we can conclude that $m^{k}$ and $c_{k}$ are the $k$-derivative of the first order characteristic function and the second characteristic function at the origin respectively.

For zero mean stationary random process $\{x(n)\}$, its $k$-order moment and $k$-order cumulants are separately represented as follows.

$$
\begin{gathered}
m_{k x}\left(\tau_{1}, \tau_{2}, \ldots, \tau_{k-1}\right)=E\left[x(n), x\left(n+\tau_{1}\right), \ldots, x\left(n+\tau_{k-1}\right)\right] \\
C_{k x}\left(\tau_{1}, \tau_{2}, \ldots, \tau_{k-1}\right)=\operatorname{cum}\left[x(n), x\left(n+\tau_{1}\right), \ldots, x\left(n+\tau_{k-1}\right)\right]
\end{gathered}
$$

And the $3^{\text {rd }}$ order cumulant of observation data $x$ can be derived from formula (8).

$$
c_{3 x}(m, n)=\operatorname{cum}[x(k), x(k+m), x(k+n)]=\left.(-j)^{3} \frac{\partial^{3} \Psi\left(\omega_{1}, \omega_{2}, . \omega_{3}\right)}{\partial \omega_{1} \partial \omega_{2} \partial \omega_{3}}\right|_{\omega_{1}=\omega_{2}=\omega_{3}=0}
$$

where, $m, n$ denote time delay, and $k=1,2, \ldots, N$.

The bi-spectrum of $x$ is the two-dimensional Fourier transform of $3^{\text {rd }}$ order cumulant $c_{3 x}$, which is defined as follows.

$$
B_{x}\left(\omega_{1}, \omega_{2}\right)=\sum_{\tau_{1}=-\infty}^{\infty} \sum_{\tau_{k-1}=-\infty}^{\infty} c_{3 x}\left(\tau_{1}, \tau_{2}\right) e^{-j\left(\omega_{1} \tau_{1}+\omega_{2} \tau_{2}\right)}
$$

Finally, when the time delays in formula (9) are equal, that is $m=n$, bi-spectrum slice can be obtained.

$$
B(\omega)=\left.\sum_{\tau=-\infty}^{+\infty} c_{3 x}(m, n)\right|_{m=n=m} e^{-j \omega m}
$$

\subsection{Bi-spectrum slice of MFSK signals}

Fig. 1 shows the relationship between bi-spectrum and the $3^{\text {rd }}$ order cumulant. Considering the characteristics of symmetry and periodicity, bi-spectrum estimation can be performed around the triangle area where contains all required information.
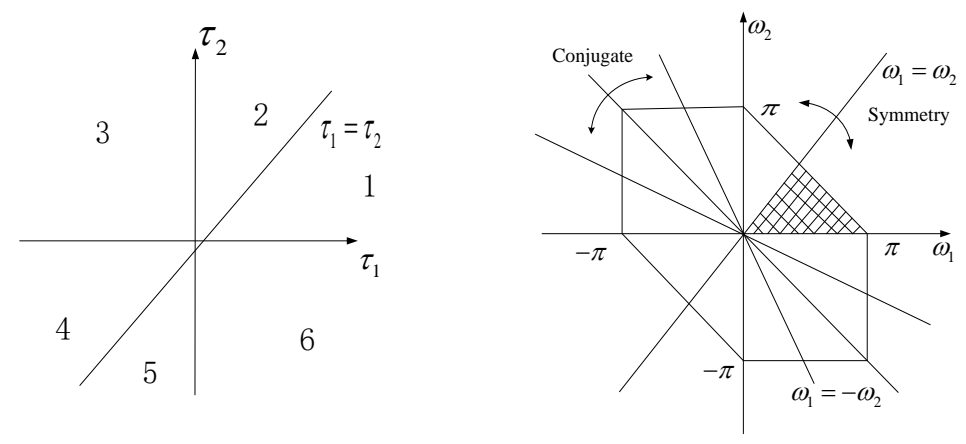

(a) Symmetric region of the $3^{\text {rd }}$ order cumulant $\quad$ (b) Symmetric region of bi-spectrum

Fig. 1. The relationship between bi-spectrum and the 3rd order cumulant 
Fig. 2 shows the bi-spectrum curves of different MFSK signals respectively. It tells us that the bi-spectrum distributions of 2FSK, 4FSK and 8FSK are different. Thus, we can use bi-spectrum characteristics to recognize different MFSK signals.

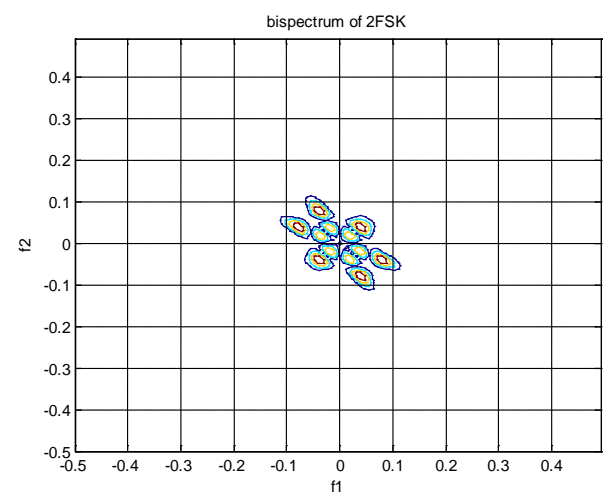

(a) Bi-spectrum of 2FSK signal

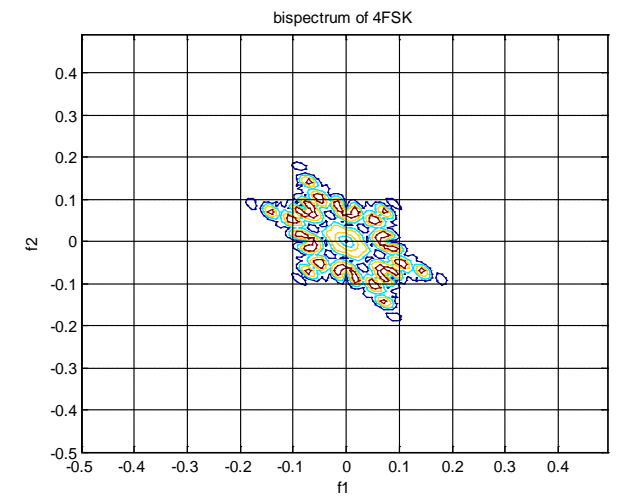

(b) Bi-spectrum of 4FSK signal

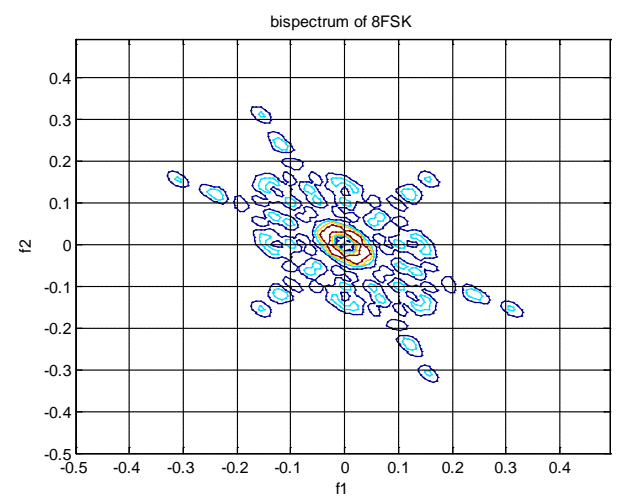

(c) Bi-spectrum of 8FSK signal

Fig. 2. Bi-spectrum curves of different MFSK signals

Fig. 3 shows the bi-spectrum slice curves of different MFSK signals respectively. It can be seen that:

(1)Gaussian noise turned into discrete, homogeneous distribution after bi-spectrum analysis, whose influence can be basically eliminated;

(2)For MFSK signals with different modulation order, the frequency and phase differences of different signals are obvious;

(3)Bi-spectrum slice can visually reflects the difference of several MFSK signals. The spectrum peak is corresponding to signal's frequency, and the number of peaks reflects signal's modulation order $M$. 


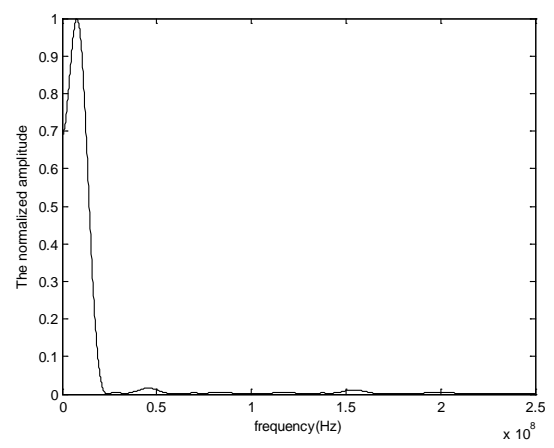

(a) Bi-spectrum slice of 2FSK signal

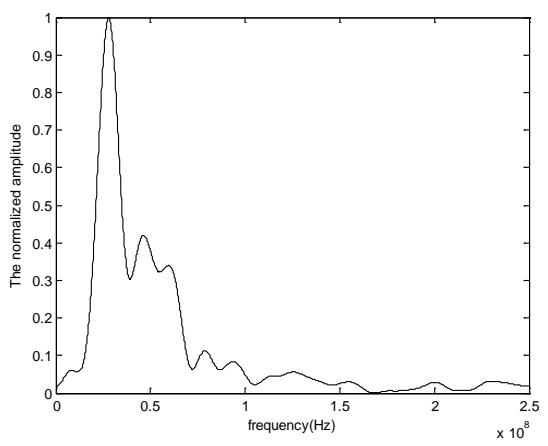

(b) Bi-spectrum slice of 4FSK signal

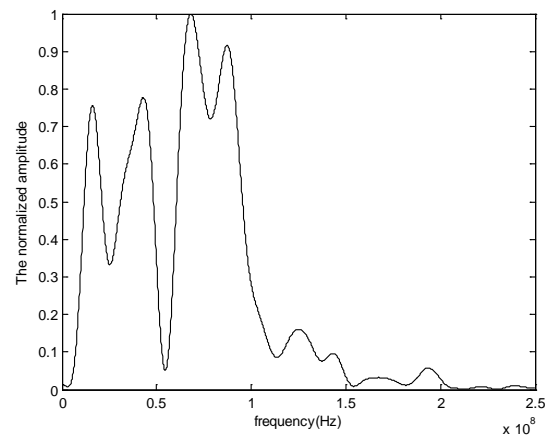

(c) Bi-spectrum slice of 8FSK signal

Fig. 3. Bi-spectrum slice of different MFSK signals

It can easily be verified in Fig. 3 that different MFSK signals have different bi-spectrum slice curves. Thus, according to the pattern that the bi-spectrum slices features of different signals are different, bi-spectrum slice information can be used as the feature for MFSK signal individual identification.

\subsection{Envelop parameter of Bi-spectrum slice}

Extracting envelope parameter of bi-spectrum slice, and establishing feature database.

For bi-spectrum slice sequence $B:\{B(1), B(2), \ldots, B(N)\}$, where $N$ is the sequence length, the envelope parameter $R$ is defined as:

$$
R=\frac{1}{\sum_{i=1}^{N}\left(B(i)-\left(\frac{1}{N} \sum_{i=1}^{N} B(i)\right)\right)^{2}}
$$

where, $\frac{1}{N} \sum_{i=1}^{N} B(i)$ represents $1^{\text {st }}$ order moment of $B(i)$, and $R$ represents the reciprocal of the variance of $B(i)$.

From formula (12), we can conclude that $R$ describes the envelope information of bi-spectrum slice sequence, which directly explains why it can be used for MFSK signal individual identification. 
Fig. 4 shows the envelop parameters $R$ of different MFSK signals under different SNRs. It can be observed that the features $R$ of different signals are obviously different even when the SNR is around $-6 \mathrm{~dB}$, which verifies the contribution of feature $R$ to MFSK signal individual identification.

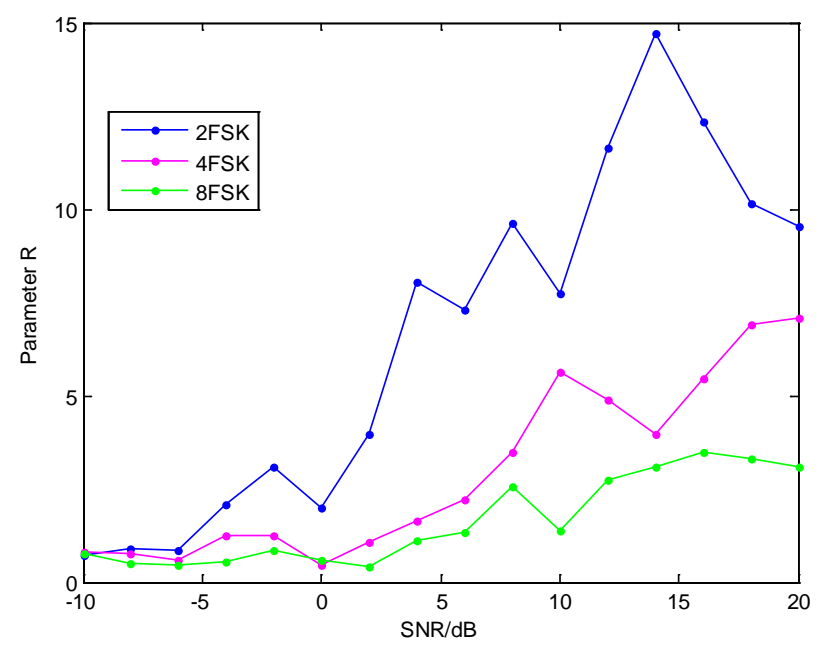

Fig. 4. Envelop parameter $R$ of MFSK signals

\section{Wavelet Low Frequency Feature Extraction}

The wavelet function refers to a function with limited length and fluctuations, which is deduced from the Fourier transform. Fourier transform analyzes signal with a fixed window, while the width of the analysis window of the wavelet transform is variable.

\subsection{Introduction of wavelet transform}

Wavelet function should satisfy the following admissibility conditions, namely the wavelet function $\psi(t)$ is energy constrained:

$$
C_{\psi}=\int_{R} \frac{|\hat{\psi}(\omega)|^{2}}{|\omega|} d \omega<\infty
$$

where, $\hat{\psi}(\omega)$ is the Fourier Transform of wavelet function $\psi(t)$.

Wavelet function that satisfies the condition in formula (13) means satisfying the condition of complete reconstruction or retaining the same resolution. Generally, $\psi(t)$ is known as basic wavelet function or mother wavelet function. To fulfill the complete reconstruction condition, $\hat{\psi}(\omega)$ should satisfy:

$$
\hat{\psi}(0)=\int_{-\infty}^{\infty} \psi(t) d t=0
$$

When using scale factor $a$ and shift factor $b$ to perform wavelet scaling and translation, a series of shifted wavelet functions with different analysis windows can be obtained.

$$
\psi_{a, b}(t)=\frac{1}{\sqrt{|a|}} \psi\left(\frac{t-b}{a}\right) \quad a, b \in R ; a \neq 0
$$


Coefficient $1 / \sqrt{|a|}$ is added in order to ensure that the energy of the shifted and scaled wavelet functions $\psi_{a, b}(t)$ remains the same with the mother wavelet function $\psi(t)$.

The continuous wavelet transform is represented as:

$$
W f(a, b)=\left\langle f, \psi_{a, b}\right\rangle=\frac{1}{\sqrt{|a|}} \int_{-\infty}^{\infty} f(t) \psi^{*}\left(\frac{t-b}{a}\right) d t
$$

\subsection{The first time wavelet transform of MFSK signals}

When the analyzed and wavelet analysis window are at the same symbol period, the first time wavelet transform of MFSK signals is:

$$
\begin{aligned}
C W T_{F S K}(a, \tau) & =\frac{1}{\sqrt{a}} \int_{-\infty}^{\infty} s(t) \psi^{*}\left(\frac{t-\tau}{a}\right) d t \\
& =\frac{1}{\sqrt{a}} \int_{-1 / 2}^{1 / 2} A e^{j\left(\omega_{i}(a t+\tau)+\varphi_{i}\right)} e^{j \omega_{c}(a t+\tau)} \operatorname{haar}(t) d(a t+\tau) \\
& =\frac{A}{\sqrt{a}}\left(\int_{-a / 2}^{0} e^{j\left(\omega_{i}(s+\tau)+\varphi_{i}\right)} e^{j \omega_{c}(s+\tau)} d s-\int_{0}^{a / 2} e^{j\left(\omega_{i}(s+\tau)+\varphi_{i}\right)} e^{j \omega_{c}(s+\tau)} d s\right) \\
& =-j \frac{4 A}{\sqrt{a}\left(\omega_{c}+\omega_{i}\right)} e^{j\left(\omega_{c} \tau+\varphi_{i}\right)} \sin ^{2}\left(\frac{a\left(\omega_{c}+\omega_{i}\right)}{4}\right)
\end{aligned}
$$

Take absolute value of formulation (17), the expression can be changed into :

$$
C W T_{F S K}(a, \tau)=\frac{4 A}{\sqrt{a}\left(\omega_{c}+\omega_{i}\right)} \sin ^{2}\left(\frac{a\left(\omega_{c}+\omega_{i}\right)}{4}\right)
$$

When the analyzed and wavelet analysis window are at the adjacent symbol period, the first time wavelet transform of MFSK signals is:

$$
\begin{aligned}
C W T_{F S K}(a, \tau)= & \frac{1}{\sqrt{a}} \int_{-\infty}^{\infty} s(t) \psi^{*}\left(\frac{t-\tau}{a}\right) d t \\
= & \frac{1}{\sqrt{a}} \int_{-1 / 2}^{1 / 2} A e^{j\left(\omega_{i}(a t+\tau)+\varphi_{i}\right)} e^{j \omega_{c}(a t+\tau)} \operatorname{haar}(t) d(a t+\tau) \\
= & \frac{A}{\sqrt{a}}\left(\int_{-a / 2}^{d} e^{j\left(\omega_{i}(s+\tau)+\varphi_{i}\right)} e^{j \omega_{c}(s+\tau)} d s+\int_{d}^{0} e^{j\left(\omega_{i+1}(s+\tau)+\varphi_{i+1}\right)} e^{j \omega_{c}(s+\tau)} d s\right. \\
= & \left.-j \frac{A}{\omega_{c} \sqrt{a}} e^{j / 2} e^{j\left(\omega_{i+1}(s+\tau)+\varphi_{i+1}\right)} e^{j \omega_{c}(s+\tau)} d s\right) \\
& +e^{j\left(\omega_{i+1}-\omega_{i}\right) \tau}\left(2-e^{j\left(\omega_{c}+\omega_{i}\right) d}-e^{-j\left(\omega_{c}+\omega_{i}\right) a / 2}\right)
\end{aligned}
$$

Take absolute value of formulation (19), the expression can be changed into: 


$$
\begin{aligned}
C W T_{F S K}(a, \tau) & =\frac{A}{\omega_{c} \sqrt{a}} \mid\left(e^{j\left(\omega_{c}+\omega_{i}\right) d}-e^{-j\left(\omega_{c}+\omega_{i}\right) a / 2}\right) \\
& +e^{j\left(\omega_{i+1}-\omega_{i}\right) \tau}\left(2-e^{j\left(\omega_{c}+\omega_{i+1}\right) d}-e^{-j\left(\omega_{c}+\omega_{i+1}\right) a / 2}\right) \mid
\end{aligned}
$$

It's apparent in formula (18) (20) that the amplitude of wavelet transform, which has sudden changes, is dependent on amplitude, frequency and phase of adjacent element.

Fig. 5 shows the instantaneous frequency of different MFSK signals after the first time wavelet transform, and the mutation frequency can be observed clearly.
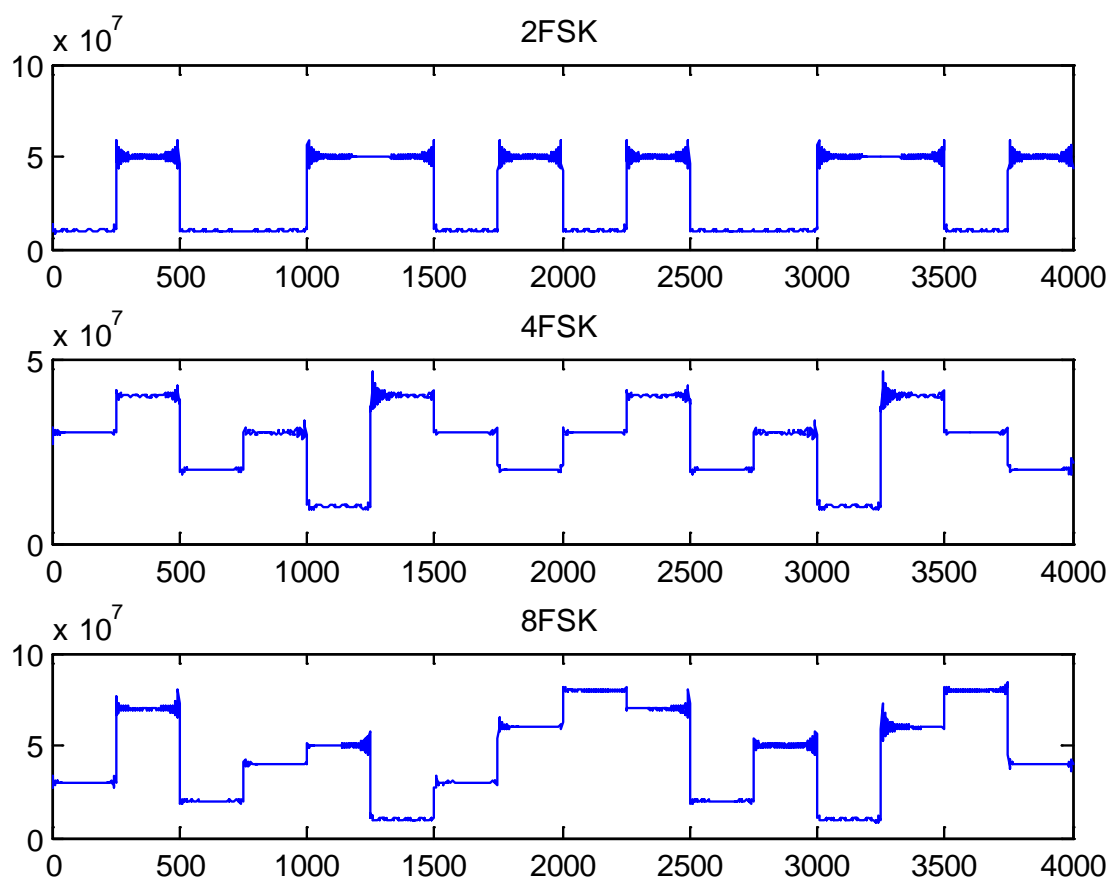

Fig. 5. The instantaneous frequency after the first time wavelet transform

The instantaneous frequency amplitude of MFSK signals after wavelet transform can be approximated as followed:

$$
x(t)=\sum_{i} A_{i} u\left(t-i T_{s}\right)+n(t)
$$

where, $A_{i}(i=2,4$, or 8$)$ is instantaneous frequency amplitude, $n(t)$ is noise frequency, $T_{s}$ is element cycles.

\subsection{The secondary wavelet transform of MFSK signals}

The secondary wavelet transform of MFSK signal is written as:

$$
c(t)=\frac{1}{\sqrt{a}} \sum_{i}\left|A_{i}-A_{i+1}\right| \delta\left(t-i T_{s}\right)
$$

Fig. 6 shows the instantaneous frequency of different MFSK signals after the secondary wavelet transform. It is obvious that the amplitude, mutation location and distribution of the wavelet coefficients are different for different MFSK signals. Thus, the distribution feature of 
the secondary wavelet transform can be used as the foundation for MFSK signal individual identification.
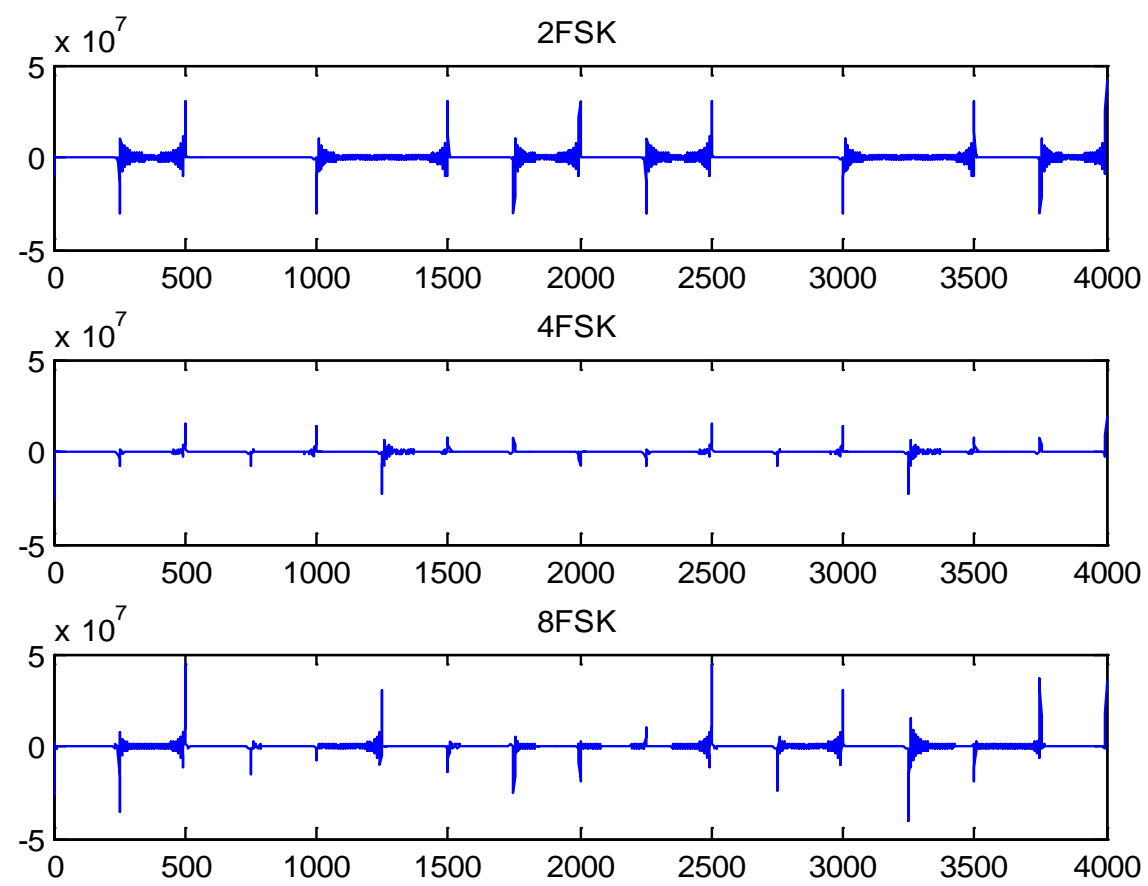

Fig. 6. The instantaneous frequency after the secondary wavelet transform

It is clear in Fig. 6 that different MFSK signals have different instantaneous frequencies of the secondary wavelet transforms. Thus, according to the pattern that the low frequency wavelet features of different signals are different, low frequency wavelet information can be applied as the feature for MFSK signal individual identification.

\subsection{Energy variance of the secondary wavelet transform coefficients}

Extracting energy variance of the secondary wavelet transform coefficients, and establishing feature database.

The computation expression of energy variance of the secondary wavelet transform coefficients $\sigma_{c}^{2}$ is defined as:

$$
\sigma_{c}^{2}=\sum_{i=1}^{L}\left(c^{2}(i)-\left(\frac{1}{L} \sum_{i=1}^{L} c^{2}(i)\right)\right)^{2}
$$

where, $c:\{c(1), c(2), \ldots, c(L)\}$ are the secondary wavelet transform coefficients.

Fig. 7 shows the energy variance of low frequency wavelet coefficients of different MFSK signals under different SNRs. are different. It can be observed that the features $\sigma_{c}^{2}$ of different signals are obviously different even when the SNR is around $-6 \mathrm{~dB}$, which verifies the contribution of feature $\sigma_{c}^{2}$ to MFSK signal individual identification. 


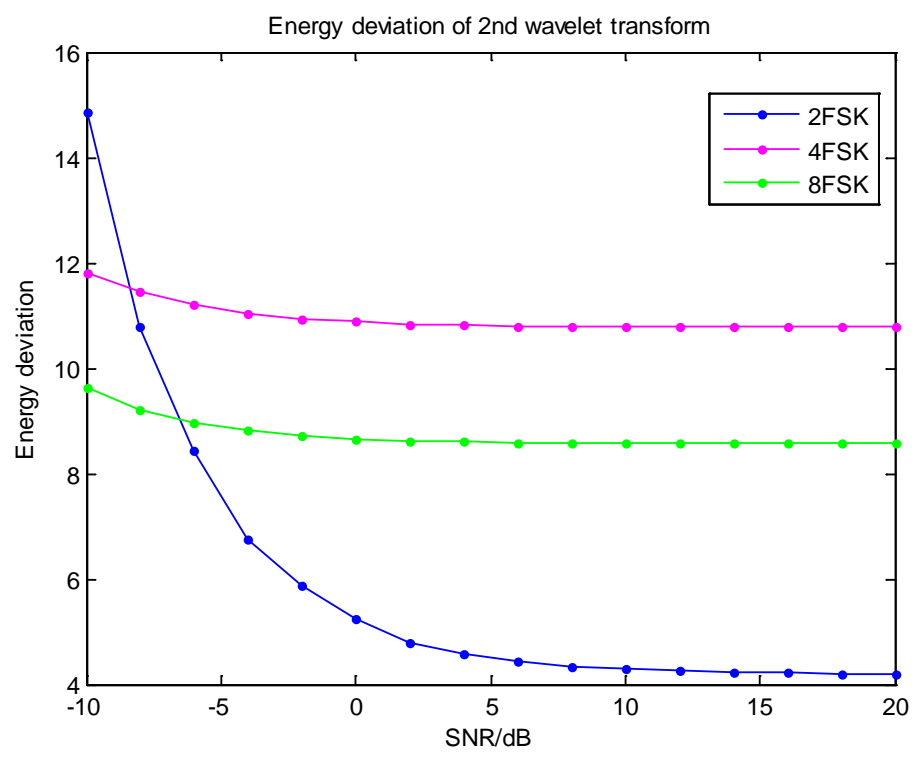

Fig. 7. Energy variance of the secondary wavelet coefficients

From Fig. 4 and Fig. 7, we can see that the envelope parameter of bi-spectrum slice and the energy variance of the secondary wavelet transform coefficients vary distinctly under high SNR, whereas, the envelope parameter of bi-spectrum slice is unstable under low SNR. Therefore, it is urgent to fuse these two features to achieve MFSK signal individual identification under low SNR.

\section{Feature Fusion under Low SNR Conditions}

As single feature individual recognition scheme has its instability to a certain extent because of random interruption, this article achieves a two-dimensional feature fusion of bi-spectrum slice envelop parameter and low frequency wavelet coefficients by introducing the idea of information fusion, which ensures the high recognition rate and robustness for MFSK signal individual identification under low SNR.

In this paper, a two-dimensional feature fusion algorithm is put forward to identify MFSK signal individual identification. It combines the advantages of bi-spectrum analysis and wavelet analysis for signal processing, which combines bi-spectrum slice envelop parameter with low frequency wavelet coefficients of received signal to realize individual identification.

Fig. 8 shows the diagrammatic sketch of MFSK signal individual identification.

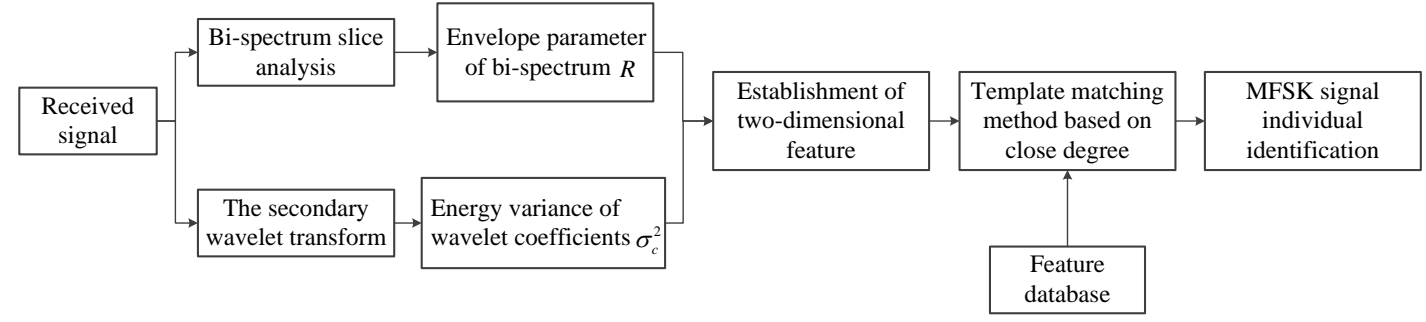

Fig. 8. The schematic of MFSK signal identification 
As we can see from Fig. 8, the specific procedure of MFSK signal individual identification can be summarized as follows.

(1) Get the Bi-spectrum of observed data and analyze the Bi-spectrum slice curve. Then, extract envelop parameter of bi-spectrum slice as the first feature;

(2) Get the secondary wavelet transform of observed data, and calculate its energy variance as the second feature;

(3) Establish two-dimensional feature of received signal;

(4) Introduce a template matching method based on close degree to compare the two-dimensional feature of received signal and feature database, and accomplish the MFSK signal individual identification.

A template matching method based on close degree is adopted to verify the validity of fused characteristics for MFSK signal individual identification.

Assume that there are $N$ categories of MFSK signals in the emitter recognition system. We can define the two-dimensional feature distance between the received signal and the feature database.

$$
D_{i}=\sum_{j=1}^{2}\left|A_{i j}-c_{j}\right|
$$

where, $c_{j}$ is the $j^{\text {th }}(j=1,2)$ feature of the received signal, and $A_{i j}$ is the $j^{\text {th }}$ feature of the $i^{\text {th }}(i=1,2, \ldots, N)$ signal in feature database.

Ulteriorly, the close degree between the received signal and the $i^{\text {th }}$ signal in feature database can be derived.

$$
N(i)=1-\sqrt{\frac{D i}{\sum_{i} D i}}
$$

When the received signal has the greatest close degree with one kind of MFSK signal in feature database, the type of received signal can be determined.

\section{Experimental Classification Results and Analyses}

In the simulation environment with matlab R2011b, we give two experiments about the novel feature fusion algorithm of MFSK signal individual identification. One is signal individual identification with same parameters and different number of $M$, and the other is signal individual identification with same number of $M(M=4)$ and different parameters.

\subsection{Experiment 1: Identification of different MFSK signals}

Parameters settings are shown in Table 1. It can be seen in Table 1 that simulation signals have different modulation types.

\begin{tabular}{|c|c|c|c|c|c|c|}
\hline & $\begin{array}{l}\text { Amplitude } \\
\text { A }\end{array}$ & $\begin{array}{r}\text { Sampling } \\
\text { frequency } \\
f_{s}(\mathrm{MHz})\end{array}$ & $\begin{array}{l}\text { Symbol rate } \\
R_{b}=1 / T_{s} \text { (us) }\end{array}$ & $\begin{array}{l}\text { Carrier } \\
\text { frequency } \\
f_{c}(\mathrm{MHz})\end{array}$ & $\begin{array}{l}\text { Signaling } \\
\text { frequency } \\
f(\mathrm{MHz})\end{array}$ & $\begin{array}{l}\text { Initial } \\
\text { phases }\end{array}$ \\
\hline $\begin{array}{l}\text { 2FSK } \\
\text { signal }\end{array}$ & $\overline{c 1}$ & $\overline{500}$ & $\overline{0.5}$ & $\overline{10}$ & $\begin{array}{l}f 1=10 \\
f 2=30\end{array}$ & $\overline{c 0}$ \\
\hline
\end{tabular}

Table 1. Parameters settings of experiment 1 


\begin{tabular}{|c|c|c|c|c|c|c|}
\hline $\begin{array}{l}\text { 4FSK } \\
\text { signal }\end{array}$ & 1 & 500 & 0.5 & 10 & $\begin{array}{l}\mathrm{f} 1=10 \\
\mathrm{f} 2=20 \\
\mathrm{f} 3=30 \\
\mathrm{f} 4=40\end{array}$ & 0 \\
\hline $\begin{array}{l}\text { 8FSK } \\
\text { signal }\end{array}$ & 1 & 500 & 0.5 & 10 & $\begin{array}{l}\mathrm{f} 1=10 \\
\mathrm{f} 2=20 \\
\mathrm{f} 3=30 \\
\mathrm{f} 4=40 \\
\mathrm{f} 5=10 \\
\mathrm{f} 6=20 \\
\mathrm{f} 7=30 \\
\mathrm{f} 8=40\end{array}$ & 0 \\
\hline
\end{tabular}

Fig. 9 gives the recognition accuracy of different MFSK signals, where the SNR range is $-10 \mathrm{~dB}$ to $20 \mathrm{~dB}$. And there are 1000 sample signals at each $1 \mathrm{~dB}$.

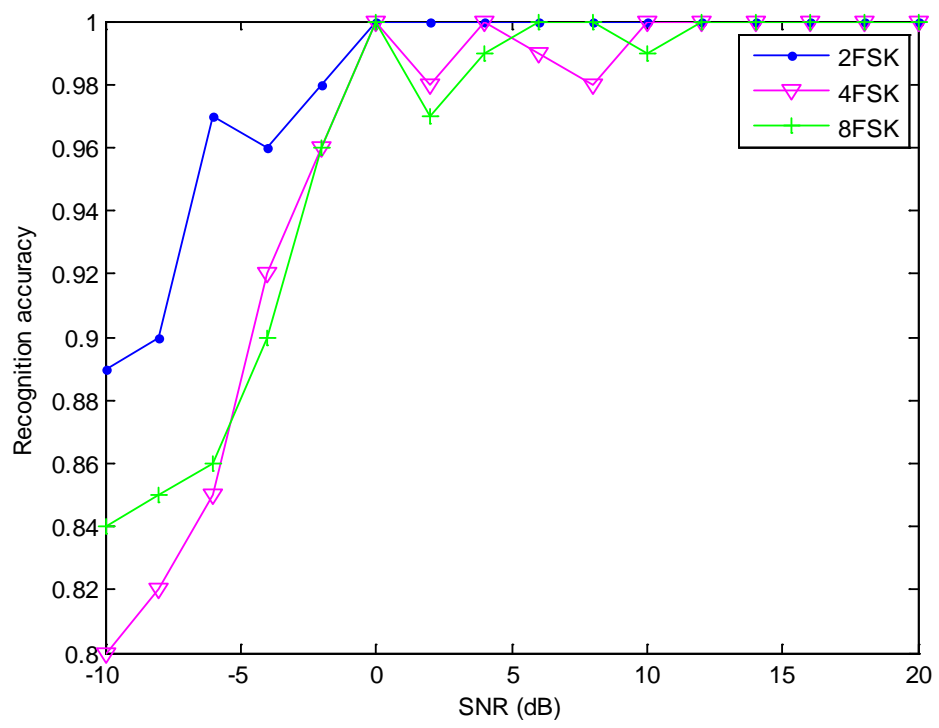

Fig. 9. Recognition accuracy of different MFSK signals

It can easily be checked in Fig. 9 that MFSK signals are completely identified when the SNR is above 0dB. Moreover, the recognition accuracy is more than $90 \%$ when the SNR is above $-4 \mathrm{~dB}$. And the recognition accuracy is more than $80 \%$ when the SNR is about $-10 \mathrm{~dB}$. Thus, the proposed algorithm realizes MFSK signal individual identification under low SNR conditions.

\subsection{Experiment 2: Identification of different 4FSK signals}

Parameters settings are shown in Table 2. It can be seen in Table 2 that signal 1 and signal 2 have distinct signaling frequency, and the symbol rate of signal 2 is the double of signal 3. 
Table 2. Parameters settings of experiment 2

\begin{tabular}{|c|c|c|c|c|c|c|}
\hline & $\begin{array}{l}\text { Amplitude } \\
A\end{array}$ & $\begin{array}{r}\text { Sampling } \\
\text { frequency } \\
f_{s}(\mathrm{MHz})\end{array}$ & $\begin{array}{l}\text { Symbol rate } \\
R_{b}=1 / T_{s} \text { (us) }\end{array}$ & $\begin{array}{l}\begin{array}{l}\text { Carrier } \\
\text { frequency }\end{array} \\
f_{c}(\mathrm{MHz})\end{array}$ & $\begin{array}{r}\text { Signaling } \\
\text { frequency } \\
f(\mathrm{MHz})\end{array}$ & $\begin{array}{c}\text { Initial } \\
\text { phases }\end{array}$ \\
\hline $\begin{array}{c}\text { Signal } \\
1\end{array}$ & $\overline{c 1}$ & 500 & $\begin{array}{ll}1.0 \\
\end{array}$ & 10 & $\begin{array}{l}\mathrm{f} 1=5 \\
\mathrm{f} 2=25 \\
\mathrm{f} 3=30 \\
\mathrm{f} 4=35\end{array}$ & 0 \\
\hline $\begin{array}{l}\text { Signal } \\
2\end{array}$ & 1 & 500 & 1.0 & 10 & $\begin{array}{l}\mathrm{f} 1=10 \\
\mathrm{f} 2=20 \\
\mathrm{f} 3=30 \\
\mathrm{f} 4=40\end{array}$ & 0 \\
\hline $\begin{array}{c}\text { Signal } \\
3\end{array}$ & 1 & 500 & 0.5 & 10 & $\begin{array}{l}\mathrm{f} 1=10 \\
\mathrm{f} 2=20 \\
\mathrm{f} 3=30 \\
\mathrm{f} 4=40\end{array}$ & 0 \\
\hline
\end{tabular}

Fig. 10 gives the recognition accuracy of different 4 FSK signals. The SNR range is $-5 \mathrm{~dB}$ to $20 \mathrm{~dB}$, and there are 1000 sample signals at each $1 \mathrm{~dB}$.

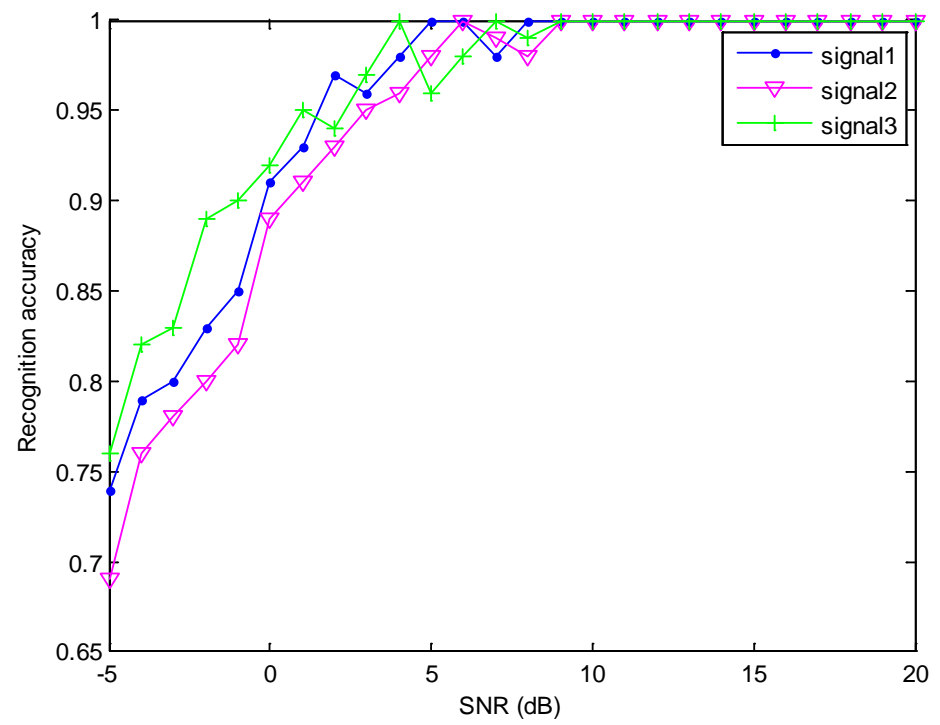

Fig. 10. Recognition accuracy of 4FSK signals

In Fig. 10, the average recognition accuracy reached 90\% when the SNR is above 3dB, which verified the effectiveness of the proposed algorithm for 4FSK signal individual identification.

Based on above two experimental results and analyses, the proposed two-dimensional feature fusion algorithm not only realizes different MFSK signal individual identification, but also achieves different 4FSK signal individual identification.

Thus, compared to MFSK signal individual identification methods with single feature, the superiority of the proposed algorithm is overt, and the significance and contribution of this paper is proved. 


\section{Conclusion}

On the basis of analyzing the characteristics of bi-spectrum analysis and wavelet transform, we propose a novel MFSK signal individual identification algorithm. The novel algorithm combines the envelope characteristic of bi-spectrum slice with energy variance of the secondary wavelet transform coefficients to recognize different MFSK signals. Theoretical analyses and computer simulation results demonstrate that this algorithm has great recognition performance with the ability to suppress noise and interference, and reaches the accurate recognition rate of more than $90 \%$ when the SNR is $-6 \mathrm{~dB}$.

In further study, as an individual identification algorithm of MFSK signal is investigated in this paper, how to apply the algorithm to signals with other modulation types has great research significance.

\section{References}

[1] Maksymyuk T, Han L, Ge X, et al., "Quasi-quadrature Modulation Method for Power Efficient Video Transmission over LTE Networks,” IEEE Transactions on Vehicular Technology, vol.63, no.5, pp.2083-2092, 2014. Article (CrossRef Link)

[2] Shi Q, Karasawa Y, “On Signal Phase Based Modulation Classification,” in Proc. of 2011 IEEE International IEEE Conference on Communications(ICC), pp.1-5, 2011. Article (CrossRef Link)

[3] Luo S E, Zhang X Y, and Luo L Y, "Subband processing based symbol-rate estimation method for MFSK signal,” in Proc. of IEEE 10th International Conference on Signal Processing (ICSP), pp.38-41, 2010. Article (CrossRef Link)

[4] Xu J F, Wang F P, and Wang Z, “Algorithm for Symbol Rate Estimation of MFSK," in Proc. of International Conference on Graphic and Image Processing, International Society for Optics and Photonics, vol.8285, 2011. Article (CrossRef Link)

[5] Yu Z, Shi Y Q, and Su W, "M-ary frequency shift keying signal classification based on discrete Fourier transform," in Proc. of Military Communications Conference, MILCOM'03, vol.2, pp.1167-1172, 2003. Article (CrossRef Link)

[6] Avci E, Avci D, "A novel approach for digital radio signal classification: Wavelet packet energy-multiclass support vector machine (WPE-MSVM)," Expert Systems with Applications, vol.34, no.3, pp.2140-2147, 2008. Article (CrossRef Link)

[7] Li J C, Li Y B, Kidera S, et al, "A robust signal recognition method for communication system under time-varying SNR environment,” IEICE Transactions on Information and Systems, vol.96, no.12, pp.2814-2819, 2013. Article (CrossRef Link)

[8] Zhang H M, Chai Y, "Digital modulation mode recognition based on multi-class classification of support vector machine,” Journal of Chongqing University (Nature Science Edition), vol.34, no.12, pp.78-81, 2013. Article (CrossRef Link)

[9] Nandi A.K, and Azzouz E.E, "Algorithms for automatic modulation recognition of communication signals,” IEEE Transactions on Communications, vol.46, no.4, pp.431-436, 1998. Article (CrossRef Link)

[10] Zhao Z, Shang J, "A new method for modulation type recognition based on the time frequency representations," in Proc. of 6th International IEEE Conference on Signal Processing, vol.1, pp.208-211, 2002. Article (CrossRef Link)

[11] Li Y B, Nie W, Ye F, et al, "A pulse signal characteristics recognition algorithm based on multifractal dimension,” Mathematical Problems in Engineering, article ID 376586, pp:1-6, 2014. Article (CrossRef Link)

[12] Dobre O A, Abdi A, Bar-Ness Y, et al, "Survey of automatic modulation classification techniques: classical approaches and new trends,” IET Communications, vol.1, no.2, pp.137-156, 2007.

Article (CrossRef Link) 
[13] Dobre O A, Rajan S, and Inkol R, “A novel algorithm for blind recognition of M-ary frequency shift keying modulation,” Wireless Communications and Networking Conference(WCNC), IEEE, pp.520-524, 2007. Article (CrossRef Link)

[14] Zhang X Y, Luo L Y, and Luo W Z, "Novel effective parameter for MPSK/MFSK classification,” Journal of Communications, vol.1, no.18, pp.108-112, 2009. Article (CrossRef Link)

[15] Li S P, Chen F C, "Recognition algorithm of digital modulation based on wavelet and high-order cumulants,” Journal of Computer Applications, vol.31, no.11, pp.2926-2928, 2011. Article (CrossRef Link)

[16] Gao J, Dong W, “A Within-class Identification Method of MFSK and MQAM Signals Based on Wavelet Transform,” Radio Engineering of China, nol.39, no. 8, pp.26-28, 2009. Article (CrossRef Link)

[17] Zeng D, Zeng X, Cheng H, et al, “Automatic modulation classification of radar signals using the Rihaczek distribution and Hough transform,” IET Radar, Sonar and Navigation, vol.6, no.5, pp.322-331, 2012. Article (CrossRef Link)

[18] Zhao C, Yang W, "Modulation Recognition of MFSK Signals Based on Multifractal Spectrum," Wireless Personal Communications, vol.72, no.4, pp.1903-1914, 2013. Article (CrossRef Link)

[19] Zhang X D, Shi Y, and Bao Z, "A new feature vector using selected bispectra for signal classification with application in radar target recognition," IEEE Transactions on Signal Processing, vol.49, no.9, pp.1875-1885, 2001. Article (CrossRef Link)

[20] Dai W, Wang Y, and Wang J, “Joint power and modulation classification using second and higher statistics," in Proc. of Wireless Communications and Networking Conference(WCNC), pp.155-158, 2002. Article (CrossRef Link)

[21] Luo L, "Comments on signal classification using statistical moments," IEEE Transactions on Communications, vol.50, no.2, pp.195, 2002. Article (CrossRef Link)

[22] Beidas B F, Weber C L, “Asynchronous classification of MFSK signals using the higher order correlation domain,” IEEE Transactions on Communications, vol.46, no.4, pp.480-493, 1998. Article (CrossRef Link)

[23] Tan X H, Chen Y, "Automatic digital modulation recognition based on combined feature parameters,” Systems Engineering and Electronics, vol.33, no.12, pp.2732-2736, 2011. Article (CrossRef Link)

[24] Qian T, Vai M I, and Xu Y, “Wavelet analysis and applications,” Springer Science \& Business Media, vol.56, no.1-2, pp.231-262, 2007. Article (CrossRef Link)

[25] He C, Han Z D, Chen L, et al, "Identification of MFSK Signals Based on Approximate Wavelet Ridge,” Journal of art force radar academy, vol.21, no.2, pp.62-64, 2007. Article (CrossRef Link)

[26] Maliatsos K, Vassaki S, and Constantinou P, "Interclass and intraclass modulation recognition using the wavelet transform,” in Proc. of IEEE 18th International Symposium on Personal, Indoor and Mobile Radio Communications(PIMRC), pp.1-5, 2007. Article (CrossRef Link) 

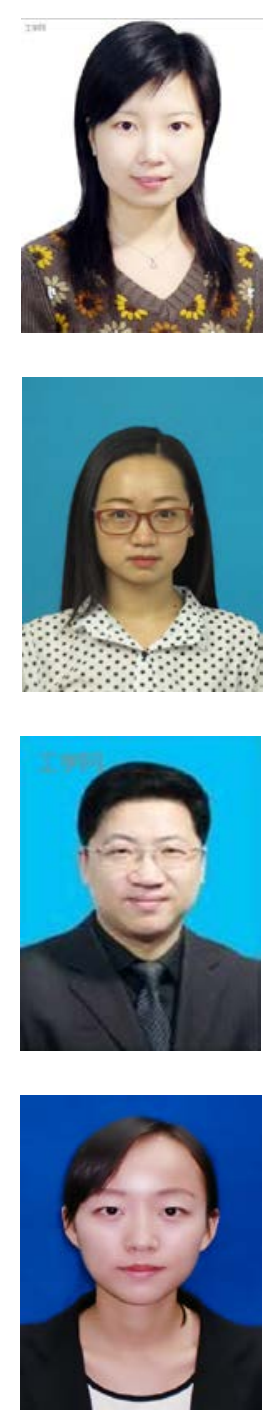

Fang Ye received the B.S. and Ph.D degrees in Electrical Information Engineering from Harbin Engineering University(HEU) in 2002 and 2006, respectively. She has been a teacher in Harbin Engineering University of China since 2002, and became an associate professor in 2007. During 2007-2008, she stayed in School of Electronics and Computer Science from University of Southampton as a visiting scholar. Now she is a IEEE member, a member of China Institute of Communications and a member of China Computer Federation.

Jie Chen received the B.S. and M.S. degrees in Electrical Information Engineering from Harbin Engineering University(HEU) in 2014 and 2016, respectively. Now, she is puresuing a Ph.D degree in information and communication engineering at HEU. Her current research intests are information fusion, integrated navigation algorithm and signal identification.

Yibing Li received the B.S. , M.S. and Ph.D degrees in Harbin Marine engineering college, Harbin engineering university in 1989, 1997 and 2003, respectively. He has been a teacher in Harbin Engineering University of China since 1989, and became an professor in 2004. During 2007-2008, he stayed in the University of Hong Kong Electronic Engineering lab as a visiting scholar. Now he is a IEEE member, a senior member of China Institute of Communications and a senior member of China Computer Federation.

Juan Ge received the B.S. and M.S. in Electrical Information Engineering and Communication and Information System from Harbin Engineering University(HEU) in 2011 and 2014, respectively. During 2012-2013, she was in University of Electro-Communication (UEC) as an exchanged student. Now she is working at Southwest China research institute of electronic equipment. 